\title{
Mathematics of Crystallographic Texture in Martensitic and Related Transformations
}

\author{
H. K. D. H. Bhadeshia, S. Kundu ${ }^{*}$ and H. Abreu* \\ University of Cambridge, Materials Science and Metallurgy, Cambridge, U.K. \\ ${ }^{*}$ TATA Steel, Research and Development Division, Jamshedpur, India \\ -Universidade Federal do Cearà, Engenharia Metalàrgica e de Materiais, Fortaleza, Brasil
}

\begin{abstract}
This paper is an introduction to the mathematical estimation of the crystallographic texture and microstructure resulting from the displacive transformation of austenite in steels, under the influence of an externally applied system of stresses. It begins with an introduction to the problem, a description of the phenomenological theory of martensite crystallography, and the application of this theory along with a variant selection criterion to determine the texture due to solid-state, displacive transformation. It is demonstrated that there remain difficulties which make a complete closure between theory and experiment unlikely. Progress is needed in relating the chemical and mechanical driving forces for phase transformation to the evolution of overall volume fractions of different crystallographic variants.
\end{abstract}

\section{Introduction}

In general usage the term texture refers to the feel of a material or object, due to some sort of a pattern within the material. Crystallographic texture is said to exist in a polycrystalline material when the distribution of crystal orientations is not random relative to some frame of reference. An understanding of texture can help relate singlecrystal properties to those of aggregates of crystals [1,2]. The texture can also be used to engineer the properties of grain boundaries to optimise them for corrosion resistance, magnetic anisotropy etc.

In a displacive transformation, the crystal structure of the parent is deformed into that of the product without the need for any diffusion. Since this involves the co-ordinated motion of atoms, the product phase is confined within the boundaries of the original austenite grain in which it nucleated; there is always therefore a fixed orientation relationship between the parent and the product phases. In this simplified scenario, it becomes possible in principle to rigorously calculate the transformation texture. To do this requires an understanding of the classical theory of martensite crystallography $[3,4]$. We begin therefore with an introduction to this theory. 


\section{Crystallographic Theory of Martensite}

\section{Structure of the Interface}

Any process which contributes to the formation of martensite cannot rely on assistance from thermal activation. There must therefore exist a high level of continuity across the interface, which must either be coherent or semi-coherent. A stress-free fully coherent interface is impossible for the austenite $(\gamma)$ to martensite $\left(\alpha^{\prime}\right)$ transformation since the lattice deformation BR is an invariant-line strain. The semi-coherent interface must be such that the interfacial dislocations can glide as the interface moves (climb is not permitted). It follows that the Burgers vectors of the interface dislocations must not lie in the interface plane unless the dislocations are screw in character.

There is an additional condition for a semi-coherent interface to be glissile. The line vectors of the interfacial dislocations must lie along an invariant-line, i.e., a line which joins the parent and product crystals without any rotation or distortion. Why is that? If there is any distortion along the dislocation line, then other dislocations are needed to accommodate that misfit. It will then be necessary to have more than one set of nonparallel dislocations in the interface. These non-parallel dislocations can intersect to form jogs which render the interface sessile.

It follows that for martensitic transformation to be possible, the deformation which changes the parent into the product must leave one or more lines invariant (unrotated, undistorted). A deformation which leaves one line invariant is called an invariant-line strain.

\section{The Shape Deformation}

The passage of a slip dislocation through a crystal causes the formation of a step where the glide plane intersects the free surface (Fig. $1 \mathrm{a}, \mathrm{b}$ ). The passage of many such dislocations on parallel slip planes causes macroscopic shear (Fig. 1 c,d). Slip causes a change in shape but not a change in the crystal structure, because the Burgers vectors of the dislocations are also lattice vectors.

During martensitic transformation, the pattern in which the atoms in the parent crystal are arranged is deformed into that appropriate for martensite, there must be a corresponding change in the macroscopic shape of the crystal undergoing transformation. The dislocations responsible for the deformation are in the $\alpha^{\prime} / \gamma$ interface, with Burgers vectors such that in addition to deformation they also cause the change in crystal structure. The deformation is such that an initially flat surface becomes uniformly tilted about the line formed by the intersection of the interface plane with the free surface. Any scratch traversing the transformed region is similarly deflected though the scratch remains connected at the $\alpha^{\prime} / \gamma$ interface. These observations, and others, confirm that the measured shape deformation is an invariant--plane strain (Fig.1 e-g) with a large shear component $(\approx 0.22)$ and a small dilatational strain $(\approx 0.03)$ directed normal to the habit plane. 


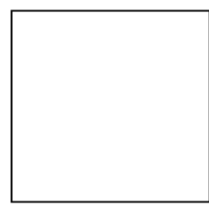

(a)

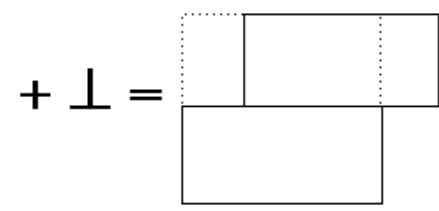

(b)

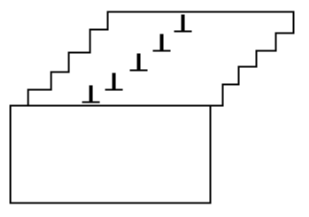

(c)

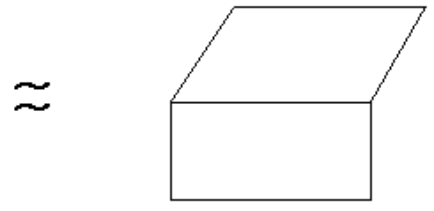

(d)

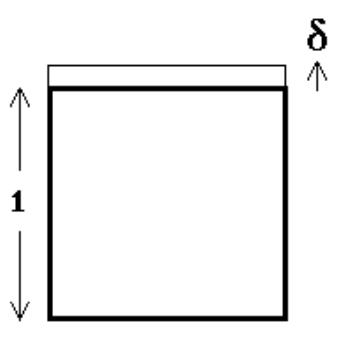

(e) uniaxial dilatation

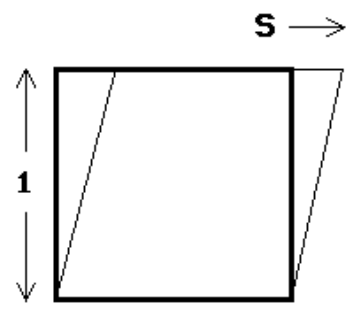

(f) shear

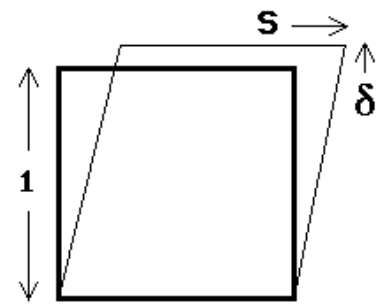

(g) IPS

Fig. 1 (a, b) Step caused by the passage of a slip dislocation. (c, d) Many slip dislocations, causing a macroscopic shear. (e) An invariant-plane strain with a uniaxial dilatation. (f) An invariant--plane strain which is a simple shear. (g) An invariant--plane strain which is the combined effect of a uniaxial dilatation and a simple shear.

\section{The Bain Strain}

Consider the displacive transformation of austenite (cubic-close packed crystal structure) to martensite (body-centred cubic or body-centred tetragonal). The change in crystal structure is achieved by a homogeneous deformation known as the Bain strain $\mathbf{B}$, which although proposed in 1924 [5] has stood the test of time as the pure deformation which achieves the desired change with the smallest strains [6]. The diagonal terms of the $3 \times 3$ matrix $\mathbf{B}$ are given by $\mathrm{a}_{\alpha^{\prime}} / \mathrm{a}_{\gamma}, \sqrt{2} \mathrm{a}_{\alpha^{\prime}} / \mathrm{a}_{\gamma}$ and $\sqrt{ } 2 \mathrm{a}_{\alpha^{\prime}} / \mathrm{a}_{\gamma}$, whereas the remaining terms are zero when $\mathbf{B}$ is defined relative to the principal axes. $\mathrm{a}_{\alpha^{\prime}}$ and $\mathrm{a}_{\gamma}$ are the lattice parameters of martensite and austenite, respectively. The Bain correspondence is illustrated in Fig. 2 and implies the orientation relationship: 


$$
\begin{gathered}
\left.\left[\begin{array}{lll}
1 & -1 & 0
\end{array}\right]_{\gamma}|| \begin{array}{lll}
1 & 0 & 0
\end{array}\right]_{\alpha} \\
\left.\left[\begin{array}{lll}
1 & 1 & 0
\end{array}\right]_{\gamma}|| \begin{array}{lll}
0 & 1 & 0
\end{array}\right]_{\alpha}
\end{gathered}
$$

This orientation is not observed experimentally because the strain energy associated with B would be too large, several $\mathrm{kJ} \mathrm{mol}^{-1}$ [7], which is far in excess of the chemical driving force for transformation [8].

Furthermore, the Bain strain does not satisfy the minimum requirement of martensitic transformation, that the deformation must leave one line invariant in order to ensure sufficient coherency in the $\gamma / \alpha$ ' interface to enable it to move without diffusion $[3,4,9$ 11]. This can be seen in Figs.3a, b; the austenite is represented as a sphere which, as a result of the Bain strain $\mathbf{B}$, is deformed into an ellipsoid of revolution which represents the martensite. There are no lines which are left undistorted or unrotated by $\mathbf{B}$. There are no lines in the $\left(\begin{array}{lll}0 & 0 & 1\end{array}\right)_{\gamma}$ plane which are undistorted. The lines $a b$ and $c d$ are undistorted but are rotated to the new positions $a^{\prime} b^{\prime}$ and $c^{\prime} d^{\prime}$. Such rotated lines are not invariant. However, the combined effect of the Bain strain $\mathbf{B}$ and the rigid body rotation $\mathbf{R}$ is indeed an invariant-line strain (ILS) because it brings $c d$ and $c^{\prime} d{ }^{\prime}$ into coincidence (Fig. 3c). This is the reason why the observed irrational orientation relationship differs from that implied by the Bain strain. Indeed, the rotation required to convert $\mathbf{B}$ into an invariant line strain precisely corrects the Bain orientation into that which is observed experimentally.

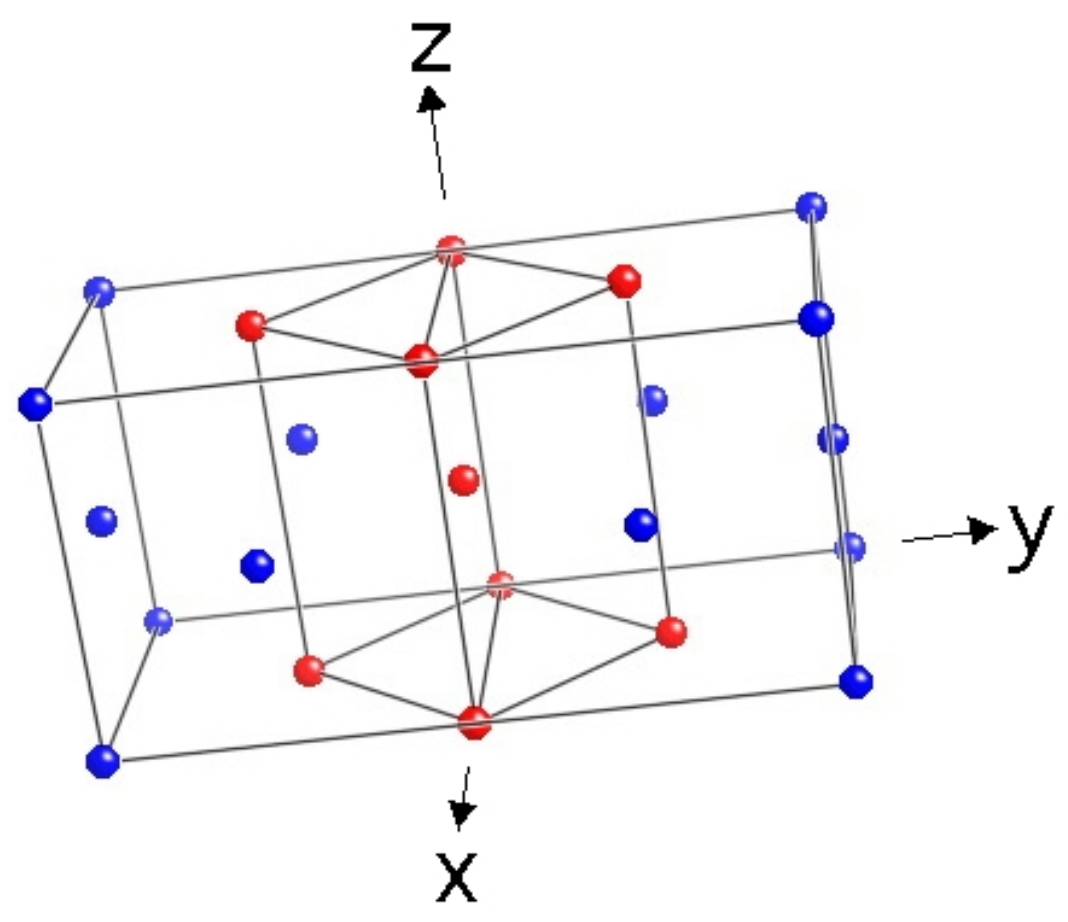


Figure 2: Two face-centred cubic unit cells of austenite, together with a body-centred tetragonal cell of austenite. The Bain strain (not illustrated here) involves a compression of the body--centred tetragonal cell of austenite along $z$ and a uniform expansion on the $x-y$ plane.

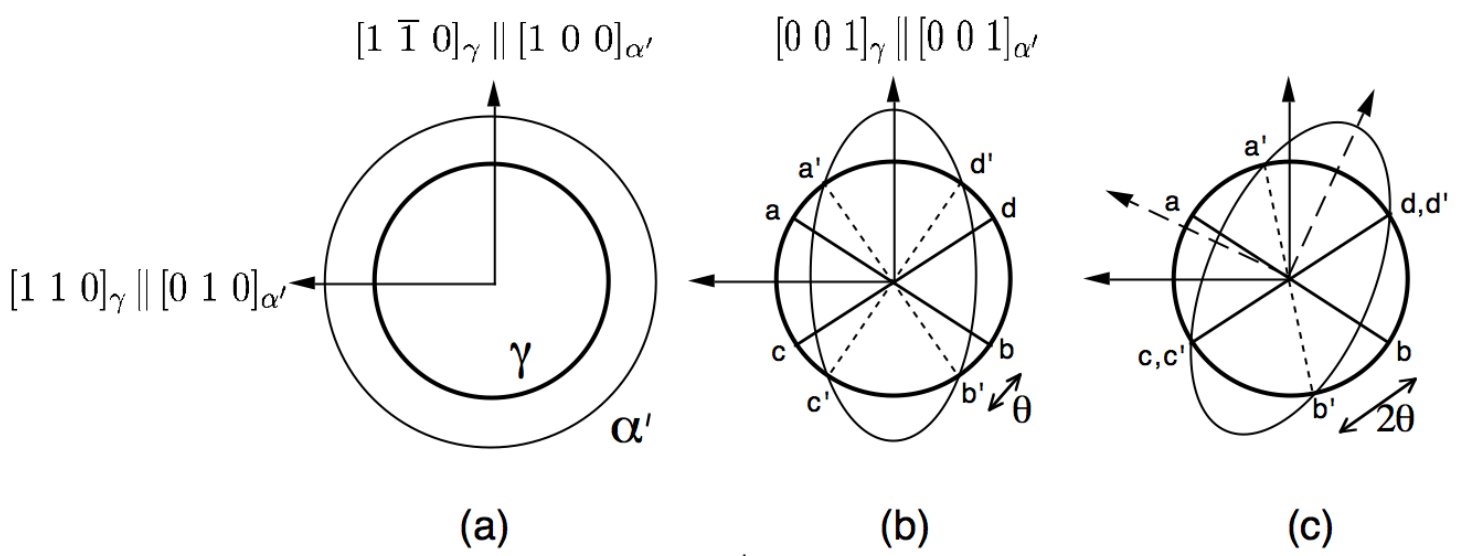

Fig. 3: (a) and (b) show the effect of the Bain strain on austenite, which when undeformed is represented as a sphere in three-dimensions. The strain transforms it to an ellipsoid of revolution. (c) shows the ILS obtained by combining the Bain strain with a rigid body rotation through an angle $\theta$.

It is now possible to reach some conclusions regarding orientation relationships in relation to transformation textures, whether these are for displacive or reconstructive phase change $[12,13]$.

- The Bain orientation relationship does not exist. It is not appropriate to use this in calculating transformation texture. Any favourable conclusions reached based on the Bain orientation $[14,15]$ must be regarded as fortuitous (Hutchinson 2005).

- The Bain deformation is an incomplete description of the transformation strain and hence should not form the basis for variant selection [16].

As stated above, the combination BR predicts the exact orientation relationship which is irrational. However, it is often assumed in texture analysis that the orientation relationship between the austenite and martensite is that due to Kurdjumov-Sachs (KS) or Nishiyama-Wasserman (NW) [13,17-19], but it has been known for some time that the true relation must be irrational $[3,4,9,11]$. Although the difference between this irrational and assumed orientation may seem less than a few degrees, it is vital because the assumed orientations do not in general lead to an invariant--line between the parent and product lattices. The existence of an invariant line is an essential requirement for martensitic transformation to occur. It is not surprising therefore, that Nolze [20] in his experimental study of several hundred thousand $\gamma / \alpha^{\prime}$ orientation relations, found detailed deviations from assumed Kurdjumov-Sachs etc. orientations. 
Much is often made of the fact that there are 24 variants of KS and only 12 of NW. However, if the actual irrational orientation is used then there will always be 24 variants.

\section{Phenomenological Solution}

We have seen that there is no rotation which can make $\mathbf{B}$ into an invariant-plane strain since this would require two non-parallel invariant-lines. It follows that austenite cannot be transformed into martensite by a homogeneous strain which is an IPS. And yet, the observed shape deformation leaves the habit plane undistorted and unrotated, i.e., it is an invariant-plane strain.

The phenomenological theory of martensite crystallography solves this remaining problem (Fig. 4). The Bain strain converts the structure of the parent phase into that of the product phase. When combined with an appropriate rigid body rotation, the net homogeneous lattice deformation RB is an invariant-line strain (step $a$ to $c$ in Fig. 4). However, the observed shape deformation is an invariant-plane strain $\mathbf{P}_{1}$ (step $a$ to $b$ in Fig. 4), but this gives the wrong crystal structure. If a second homogeneous shear $\mathbf{P}_{2}$ is combined with $\mathbf{P}_{1}$ (step $b$ to $c$ ), then the correct structure is obtained but the wrong shape since

$$
\mathbf{P}_{1} \mathbf{P}_{2}=\mathbf{R B}
$$

These discrepancies are all resolved if the shape changing effect of $\mathbf{P}_{2}$ is cancelled macroscopically by an inhomogeneous lattice-invariant deformation, which may be slip or twinning as illustrated in Fig. 4.

The theory explains all the observed features of the martensite crystallography. The orientation relationship is predicted by deducing the rotation needed to change the Bain strain into an invariant-line strain. The habit plane does not have rational indices because the amount of lattice-invariant deformation needed to recover the correct the macroscopic shape is not usually rational. The theory predicts a substructure in plates of martensite (either twins or slip steps) as is observed experimentally. The transformation goes to all the trouble of ensuring that the shape deformation is macroscopically an invariant-plane strain because this reduces the strain energy when compared with the case where the shape deformation might be an invariant-line strain. 


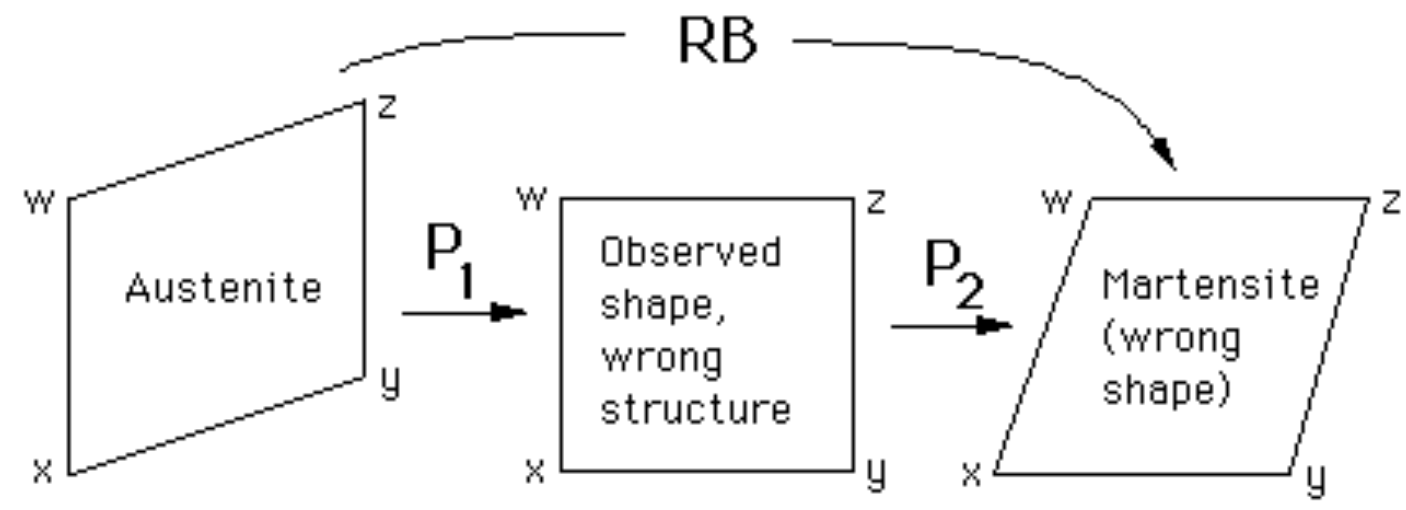

(a)

(b)

(c)

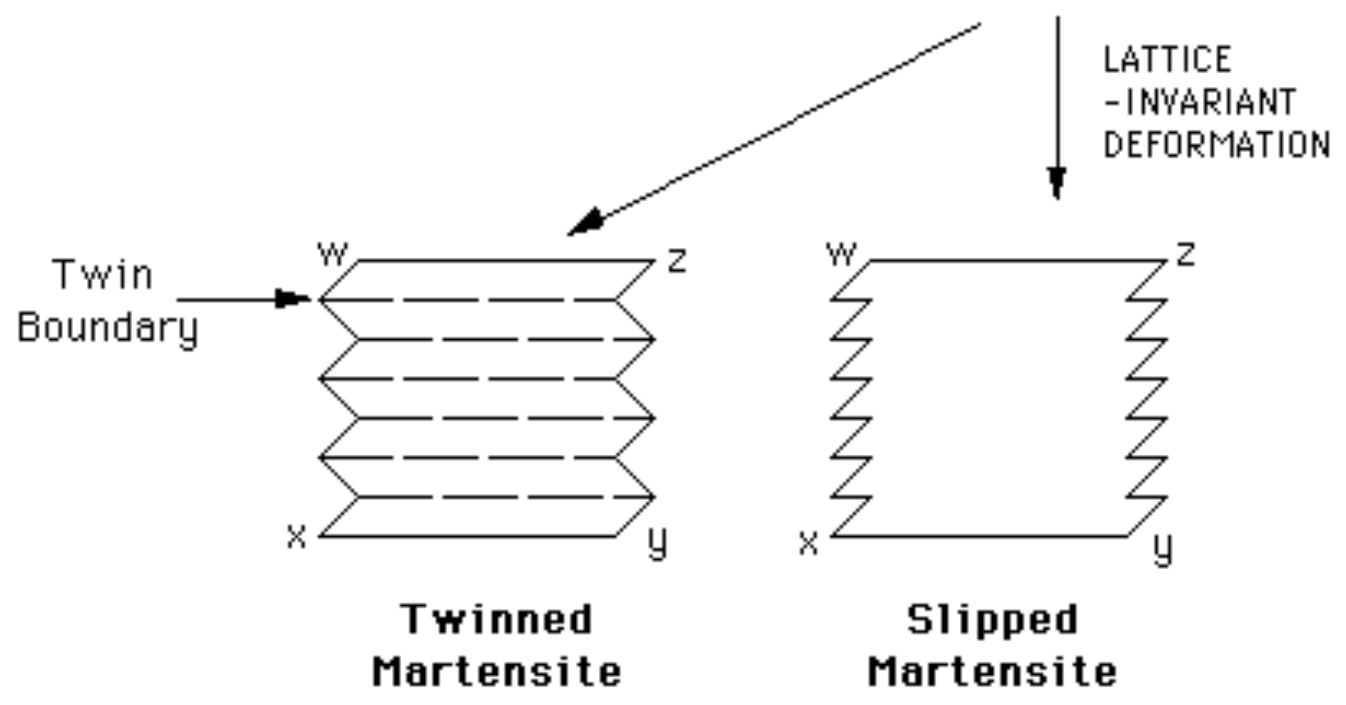

Correct macroscopic shape, correct structure

Fig. 4: The phenomenological theory of martensite crystallography

\section{The Crystallographic Set}

In order to understand how to used the crystallographic theory of martensite in estimating texture, it is important to realise that the transformation is dominated by strain energy due to the shape deformation and hence many of the crystallographic variables cannot be varied independently. Fig. 5 illustrates two crystals separated by an interface. The relative orientation of the two crystals can be described in terms of an axis-angle pair so that there are three degrees of freedom associated with the orientation relationship. One of these is the right-handed angle of rotation and the other the two independent direction cosines of the rotation axis. The interface plane, which is identified by its normal, itself can be varied whilst keeping the orientation relationship fixed. This adds another two degrees of freedom, making a total of five independent ways in which the properties of the bicrystal can be varied. 


\section{Degrees of freedom}

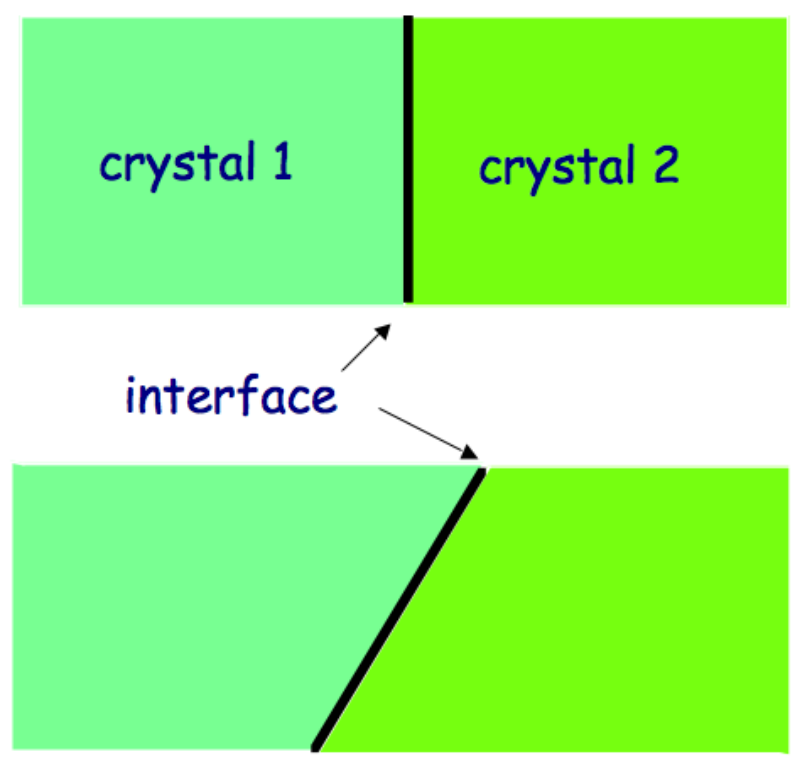

Fig. 5: The conventional degrees of freedom associated with a bicrystal containing an interface. Translations in the plane of the interface are neglected here.

This conventional description of the degrees of freedom is severely constrained in the case of displacive transformations where the orientation relationship, interface plane (habit plane), lattice-invariant deformation and shape deformation are mathematically connected by the single equation of the crystallographic theory. Changing any one of these necessarily alters all the others in this crystallographic set, an example of which is given in Fig. 6. It is not rigourous to assign an orientation and then use independent data for the shape deformation, as is sometimes done [12,19,21]. Similarly, the use of shears which are consistent with an assumed orientation relationship but not with the habit plane [22] contradicts the need for a self--consistent mathematical set. In recent work it has been incorrectly proposed that the amount of lattice-invariant shear (in the form of twin fraction) can be independently varied while keeping all the other characteristics constant [23]. 


\section{Habit plane $\mathbf{p}_{\gamma}$}

$$
\left(\begin{array}{l}
-0.168640 \\
-0.760394 \\
-0.627185
\end{array}\right)
$$

Orientation $(\gamma \mathrm{J} \alpha)$
$\left(\begin{array}{ccc}0.575191 & 0.542067 & 0.097283 \\ -0.550660 & 0.568276 & 0.089338 \\ -0.008610 & -0.131800 & 0.785302\end{array}\right)$

\section{$\left[\begin{array}{lll}1 & 0 & 1\end{array}\right]_{\gamma}||\left[\begin{array}{lll}-0.920611 & -1.062637 & 1.084959\end{array}\right]_{\alpha^{\prime}}$ (1 111$)_{\gamma} \|\left(\begin{array}{lll}0.015921 & 0.978543 & 0.971923)_{\alpha^{\prime}}\end{array}\right.$}

Shape change $(\gamma \mathrm{P} \gamma)$
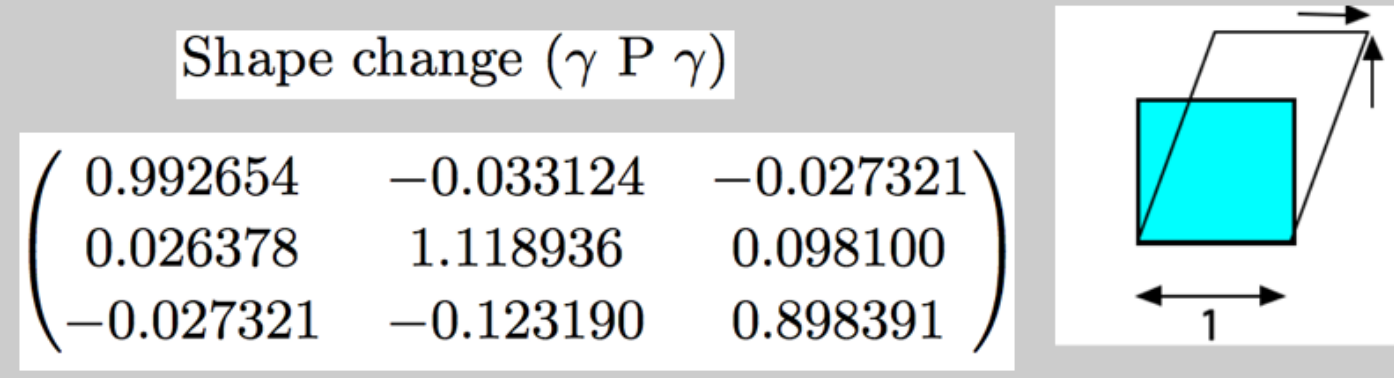

Fig. 6: An example of a complete crystallographic set describing a single plate of martensite, including the habit plane, the shape deformation and the orientation relationship (and implicitly the lattice-invariant deformation). All of these quantities are mathematically connected and cannot in general be varied independently.

\section{Variant Selection}

Displacive transformations are best regarded as deformation mechanisms which at the same time alter the crystal structure. The variant selection problem then reduces to issues similar to the selection of slip systems out of all the possibilities available during single-crystal deformation [1]. A slip system consists of a slip plane and slip direction, for example, (lll 111$)\left[\begin{array}{lll}1 & 0 & -1\end{array}\right]$ is one of 12 crystallographically equivalent systems in austenite. An applied stress is resolved on to each of the slip systems, and that which has the highest resolved shear stress is said to be activated.

By analogy, the deformation due to martensitic transformation occurs on the habit plane (unit normal p) in a displacement direction (unit vector d). The latter will not lie precisely in the habit plane because the dilatational strain due to the volume change of transformation is directed normal to the habit plane. The dominating strain is the shear parallel to the habit plane at about 0.26 . The total deformation is expressed as a $3 \times 3$ matrix $\mathbf{P}$ :

$$
(\gamma \mathbf{P} \gamma)=\mathbf{I}+m[\gamma ; \mathbf{d}]\left(\mathbf{p} ; \gamma^{*}\right)
$$


where $m$ is the magnitude of the shape deformation and $\gamma$ and $\gamma^{*}$ represent the real and reciprocal bases of the austenite. $\mathbf{P}$ thus completely defines the deformation system, and there will in general be 24 different variants.

The shape deformation $\mathbf{P}$ is an invariant--plane strain and is the experimentally observed permanent shape change caused when martensite forms.

When calculating the favoured system during slip deformation, it is the macroscopic shear on the slip plane and slip direction which determines selection through interaction with the applied stress. The detailed atomic motions (e.g. in surmounting Peierls barriers) or microscopic heterogeneities (due to the discrete nature of atoms) are irrelevant in the selection of the system. In a similar way, it is the interaction of the applied stress with $\mathbf{P}$ which determines variant selection. $\mathbf{B}$ and $\mathbf{R}$ or other factorisations of the shape deformation are incomplete descriptions of the relevant strain. The interaction energy which provides the mechanical driving force for transformation [24]:

$$
U=\sigma_{\mathrm{N}} \delta+\tau s
$$

where $\sigma_{\mathrm{N}}$ is the stress component normal to the habit plane, $\tau$ is the shear stress resolved on the habit plane in the direction of shear and $\delta$ and $s$ are the respective normal and shear strains associated with transformation. The energy $U$ can be used as a rigourous variant selection criterion when the stresses applied are less than those required to cause plasticity in the austenite prior to its transformation or when the plastic strain is not the dominant effect in variant selection [25].

The conclusions that can be reached from the discussion in this section are:

- In calculating transformation texture is is necessary to use a a self-consistent crystallographic set, rather than make independent assumptions about the orientation relationship and shape deformation as is sometimes done. The set must be such that the lattice deformation $\mathbf{B R}$ is an invariant--line strain; the analysis in [21] does not satisfy this criterion.

- The deformation due to martensitic transformation is an invariant--plane strain $\mathbf{P}$. It is this which should be used to calculate the interaction energy (variant selection) rather than, for example, the Bain strain [26].

The Patel and Cohen derivation of the interaction energy $U$ can be generalised to an arbitrary stress tensor as follows [27]. Assuming that the summation convention applies, 


$$
\begin{aligned}
\boldsymbol{\sigma}_{\boldsymbol{N}} & =\left(\sigma_{i j} \mathrm{p}_{i} \mathrm{p}_{j}\right) \mathbf{p} \\
\tau & =\left(\boldsymbol{\sigma}_{\boldsymbol{t}}-\boldsymbol{\sigma}_{\boldsymbol{N}}\right) \cdot \mathbf{e}=\sigma_{i j} \mathrm{p}_{j} \mathrm{e}_{i} \\
U & =\left[\delta \sigma_{i j} \mathrm{p}_{i} \mathrm{p}_{j}+s \sigma_{i j} \mathrm{e}_{i} \mathrm{p}_{j}\right]=\sigma_{i j} \mathrm{p}_{j}\left[\delta \mathrm{p}_{i}+s \mathrm{e}_{i}\right] \\
& =\sigma_{i j} \mathrm{p}_{j} m \mathrm{~d}_{i} \\
& \equiv \sigma_{i j} \epsilon_{i j}
\end{aligned}
$$

where $\sigma_{\mathbf{t}}$ is the traction on the plane defined by the unit habit plane normal $\mathbf{p}$ (components $\mathrm{p}_{1}, \mathrm{p}_{2}, \mathrm{p}_{3}$ ), and $\mathbf{e}$ (components $\mathrm{e}_{1}, \mathrm{e}_{2}, \mathrm{e}_{3}$ ), is the unit direction on the habit plane along which the shear component of the shape deformation is directed; $\varepsilon_{\mathrm{ij}}$ is

$$
\epsilon_{i j}=\frac{(\gamma \mathrm{P} \gamma)+\left(\gamma \mathrm{P}^{\prime} \gamma\right)}{2}-\mathrm{I}
$$

As with Patel and Cohen, these equation recognise the fact that the transformation strain is plastic.

\section{Transformation Plasticity}

Consider an arbitrary vector $\mathbf{u}$ traversing a grain of austenite prior to transformation. This vector makes an intercept $\Delta \mathbf{u}$ with a domain of austenite that eventually ends up as a plate of the displacive transformation product. It follows that the new vector $\mathbf{v}$ is given by:

$$
\mathbf{v}=\mathbf{P} \Delta \mathbf{u}+(\mathbf{u}-\Delta \mathbf{u})
$$

This is generalised for a large number of plates in many austenite grains as follows:

$$
\mathbf{v}=\sum_{k=1}^{n} \sum_{j=1}^{24} \mathbf{P}_{j}^{k} \Delta \mathbf{u}_{j}^{k}+\left(\mathbf{u}-\sum_{k=1}^{n} \sum_{j=1}^{24} \Delta \mathbf{u}_{j}^{k}\right)
$$

where $j=1 \ldots . .24$ represents the 24 crystallographic variants possible in each austenite grain, and $k=1 \ldots . . n$ represents the $n$ austenite grains traversed by the vector $\mathbf{u}$. In this scenario of a large number of plates, the various intercepts can be approximated by $f_{\mathrm{j}}^{\mathrm{k}} \mathbf{u}$ where $f_{\mathrm{j}}^{\mathrm{k}}$ is the fraction of sample transformed by variant $j$ in austenite grain $k$.

The deformation caused by a particular plate $j$ in austenite grain $k$, i.e., $\left(\gamma_{\mathrm{k}} \mathbf{P}_{\mathrm{j}} \gamma_{\mathrm{k}}\right) \equiv \mathbf{P}_{\mathrm{j}}^{\mathrm{k}}$. The remaining 23 such matrices for grain 1 of austenite can be deduced using symmetry operations and expressed in the reference frame of the sample using a similarity transformation.

Some calculations illustrating the anisotropy of strains as a function of the number of crystallographic variants of martensite allowed are illustrated in Fig. 7 for uniaxial tension and compression. That displacive transformations produce highly anisotropic 
strains when variant selection is significant has been demonstrated experimentally [2830].
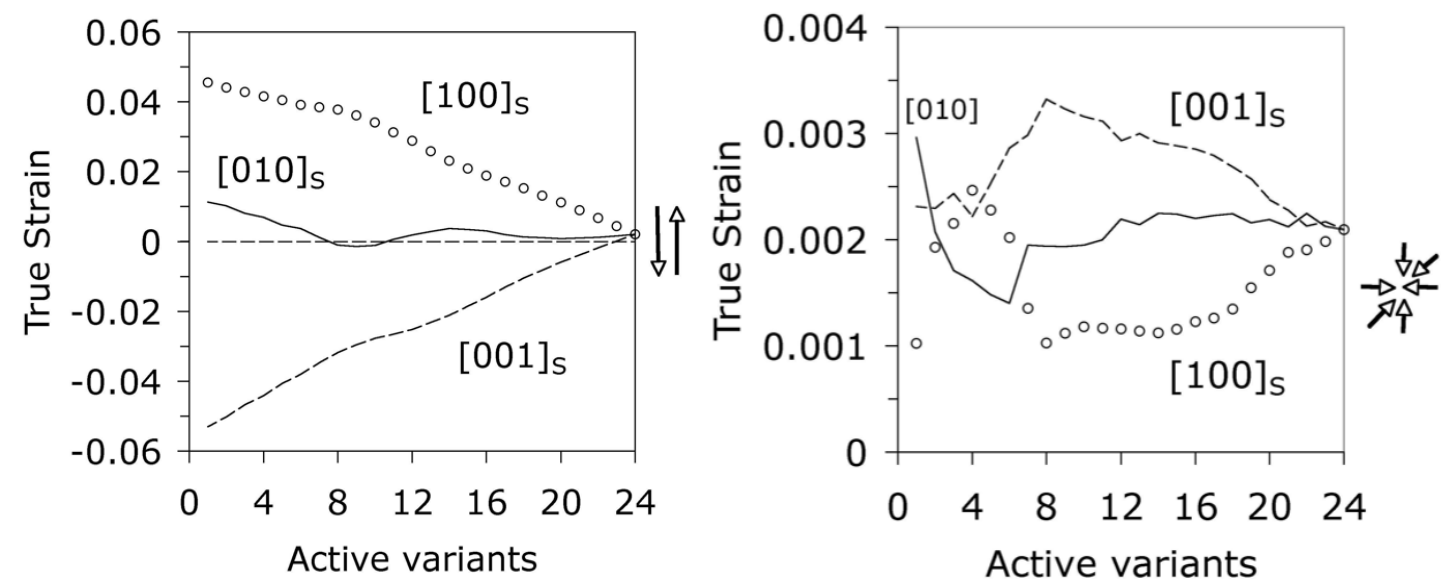

Fig. 7: (a) Transformation strain along the sample axes when a polycrystalline sample is subjected to pure shear; (b) much small strains when subjected to hydrostatic compression. After Kundu et al. [25].

An important outcome of the fact that transformation strains can be calculated using the crystallographic set of martensite is that such strains can be exploited as an alternative or supplemental method of assessing texture.

\section{The Intensity of the Texture}

The analysis of texture as described above and in the published literature, leaves open the question of the degree of variant selection as a function of the magnitude of the applied stress.

The total free energy available for transformation is the sum of chemical and mechanical components, the latter being zero in the absence of an applied stress during transformation [28]:

where $\Delta \mathrm{G}_{\mathrm{MECH}} \equiv U$.

$$
\Delta \mathrm{G}=\Delta \mathrm{G}_{\mathrm{CHEM}}+\Delta \mathrm{G}_{\mathrm{MECH}}
$$

It would be reasonable to assume that there is strong variant selection when the ratio of $\Delta \mathrm{G}_{\mathrm{MECH}} / \Delta \mathrm{G}$ is large [29]. This turns out to be the case as illustrated in Fig. 8 . There is a strong, albeit empirical, linear correlation between the ratio $\Delta \mathrm{G}_{\mathrm{MECH}} / \Delta \mathrm{G}$ and the number of most favoured variants allowed to form in each of the austenite grains [25]. This is an important observation in that it allows the extent of variant selection, and hence the transformation strains, to be calculated as a function of stress for any steel as long as the thermodynamic quantities can be estimated. Nevertheless, this clearly is an area where progress is needed from a fundamental point of view. 


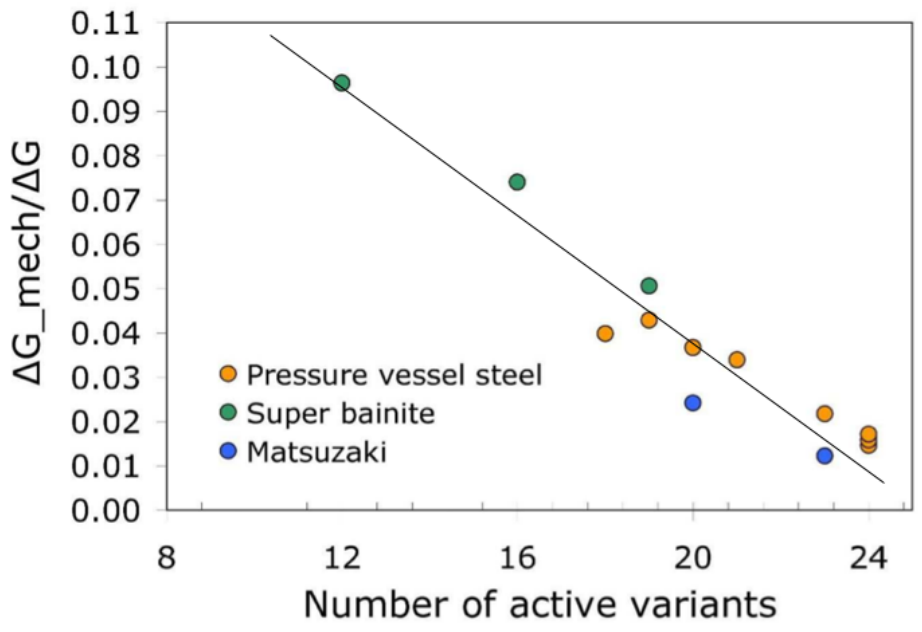

Figure 8: The ratio of the mechanical to total driving force for transformation as a function of the number of active variants for a variety of steels [25].

An additional point to emerge from this analysis is the way in which the energy $U$ is calculated. Most publications treat the problem using elasticity but the strain due to transformation is plastic so the elastic calculations underestimate $U$ by a factor of 2 . This is important when calculating the ratio of mechanical to total driving force [27].

\section{Summary}

We have seen that there are significant advantages in dealing with the crystallographic texture due to displacive transformations using the crystallographic theory of martensite along with a variant selection criterion based on the classical interaction of stress with the shape deformation. The calculation of texture at the same time leads to the estimation of the net transformation strain in any direction. This strain may be highly anisotropic depending both on the strength of the texture of the parent austenite and of the extent of variant selection. The latter in turn depends on the ratio of the mechanical driving force to the total driving force for transformation. Variant selection is minimised when the chemical driving force dominates the total. Further work is needed to develop a quantitative understanding of the intensity of the transformation texture as a function of the variant selection criterion.

\section{References}

[1] E. Schmid, W. Boas: Plasticity of Crystals (translated from the 1935 edition of Kristalplastizitaet): F. A. Hughes and Co., London, U.K., 1950.

[2] D. Dorner, S. Zaefferer, L. Lahn, D. Raabe: Journal of Magnetism and Magnetic 
Microstructure and Texture in Steels and Other Materials, editors A. Hladar, S. Suwas and D. Bhattachargee, Springer (London), 2009, pages 19-31

Materials 304 (2006) 183--186.

[3] J. K. Mackenzie, J. S. Bowles: Acta Metallurgica 2 (1954) 138--147.

[4] M. S. Wechsler, D. S. Lieberman, T. A. Read: Trans. AIME Journal of Metals 197 (1953) 1503--1515.

[5] E. C. Bain: Trans. AIME 70 (1924) 25--46.

[6] J. S. Bowles, C. M. Wayman: Metallurgical Transactions 3 (1972) 1113--1121.

[7] M. Suezawa: Materials Science and Engineering 48 (1981) 255--260.

[8] J. W. Christian: Thermodynamics and kinetics of martensite: in: G. B. Olson, M. Cohen (Eds.), International Conference on Martensitic Transformations \{ICOMAT\} '79: 1979: pp. 220--234.

[9] J. S. Bowles, J. K. MacKenzie: Acta Metallurgica 2 (1954) 129--137.

[10] J. W. Christian: Metallurgical Transactions A 13 (1982) 509--538.

[11] H. K. D. H. Bhadeshia: Geometry of Crystals: 2nd edition, Institute of Materials, 2001.

[12] W. B. Hutchinson, L. Ryde, P. S. Bate: Materials Science Forum 495--497 (2005) 1141--1149.

[13] S. Kundu, H. K. D. H. Bhadeshia: Scripta Materialia 55 (2006) 779--781.

[14] H. Miyaji, E.-I. Furubayashi: Textures and Microstructure 12 (1990) 189--197.

[15] L. Kestens, R. Decocker, R. Petro: Materials Science Forum 408--412 (2002) 1173--1178.

[16] E.-I. Furubayashi, H. Miyaji, M. Nobuki: Trans. Iron Steel Institute of Japan 27 (1987) 513--519.

[17] R. P. L. Kestens, Y. Houbaert: ISIJ International 43 (2003) 1444--1452.

[18] B. Bruckner, G. Gottstein: ISIJ International 41 (2001) 468--477.

[19] P. Bate, B. Hutchinson: Acta Materialia 48 (2000) 3183--3192.

[20] G. Nolze: Zietschrift fl"ur Metallkunde 95 (2004) 744--755.

[21] D. W. Suh, H. N. Han, S. J. Kim: ISIJ International 46 (2006) 341--343. 
[22] Y. Higo, F. Lecroisey, T. Mori: Acta Metallurgica 22 (1974) 313--323.

[23] T. Mori, E. C. Oliver, M. R. Daymond and P. J. Withers, Materials Science and Engineering A: Volume 378, Issues 1-2, 25 July 2004, Pages 479-483.

[24] J. R. Patel, M. Cohen: Acta Metallurgica 1 (1953) 531--538.

[25] S. Kundu, K. Hase, H. K. D. H. Bhadeshia: Proceedings of the Royal Society A 463 (2007) 2309--2328.

[26] H. N. Han, D. W. Suh: Acta Materialia 51 (2003) 4907--4917.

[27] S. Kundu, H. K. D. H. Bhadeshia: Scripta Materialia 57 (2007) 869--872.

[28] H. K. D. H. Bhadeshia: Possible effects of stress on steel weld microstructures: in: H. Cerjak, H. K. D. H. Bhadeshia (Eds.), Mathematical Modelling of Weld Phenomena -- II: Institute of Materials, London, U.K., 1995: pp. 71--118.

[29] H. K. D. H. Bhadeshia, S. A. David, J. M. Vitek, R. W. Reed: Materials Science and Technology 7 (1991) 686--698.

[30] A. Matsuzaki, H. K. D. H. Bhadeshia, H. Harada: Acta Metallurgica and Materialia 42 (1994) 1081--1090.

[31] J. W. Stewart, R. C. Thomson, H. K. D. H. Bhadeshia: Journal of Materials Science 29 (1994) 6079--6084. 